\title{
A neural basis for the effect of bilingual language control on cognitive control
}

Thomas Somers, Lilith Van Biesen, Esli Struys, Ghazal Mohades

Vrije Universiteit Brussel, Belgium

https://doi.org/10.36505/ExLing-2012/05/0027/000233

\begin{abstract}
It has been observed that bilinguals have an advantage on tests of cognitive control, such as the Simon task (Bialystok et al. 2004). This advantage has been attributed to the bilingual's need to constantly manage two competing languages. However, the neural correlates of this effect have not been fully understood. Although many of the networks in the brain used for cognitive control are also used for language control, it has been argued (Frühholz et al. 2010) that different types of conflicts rely on distinct neural mechanisms of conflict processing. The present study sought to explore potential overlaps in the brain circuits involved in cognitive language and control in order to provide a tentative basis for the bilingual cognitive advantage.
\end{abstract}

Key words: neurolinguistics; langanguage control; cognitive control

\section{Introduction}

The term 'cognitive control' refers to a general mechanism using different processes (attention, working memory, response selection and inhibition) in sensory, memory and motor systems to achieve a goal (Ye \& Zhou 2009).

Language control is the 'control mechanism behind languages' which assures that in a particular context the correct language is used. Several authors (Abutalebi \& Green 2007; Ye \& Zhou 2009; Garbin et al. 2010) agree that many of the networks in the brain used for cognitive control are also used for language control, these are often overly generally described as a network of frontal, parietal and subcortical structures (Ye \& Zhou 2009).

It has been observed that bilinguals have an advantage on tests of cognitive control, such as the Simon task (Bialystok et al. 2004). This advantage has been attributed to the bilingual's need to constantly manage two competing languages. However, the neural correlates of this effect have not been fully understood. Moreover, it has been argued (Frühholz et al. 2010) that different types of conflicts rely on distinct neural mechanisms of conflict processing: whereas performance on the Simon task relies on response inhibition (a stimulus-response conflict), language control relies on interference inhibition (a stimulus-stimulus conflict; see van Heuven et al. 2008).

The present study sought to explore potential overlaps in the brain circuits involved in cognitive control and language control in order to provide a tentative basis for the advantage of bilinguals on cognitive control tasks.

ExLing 2012: Proceedings of 5th Tutorial and Research Workshop on Experimental Linguistics, 27-29 August 2012, Athens, Greece 


\section{Experimental procedures}

\section{Participants}

12 simultaneous (5 females; mean age 20,51; SD 1,89) and 12 sequential (6 females; mean age 20,56 years, SD 2,12; mean AoA French 9,25 years, SD 0,87 ) healthy right-handed Dutch-French bilinguals.

\section{Tasks}

In the Simon task (Simon 1969) each trial began with a fixation cross presented for $300 \mathrm{~ms}$ followed by a red or blue square presented for a mean duration of $2200 \mathrm{~ms}$ (ISI $=0$ ) on the left or right side of the screen. The mapping of stimulus colour to response key was counterbalanced across participants. Each scan run comprised 156 trials.

The semantic categorization task consisted of 78 target animal nouns (39 Dutch, 39 French) and 78 random nouns (39 Dutch, 39 French) with mean length $=5.8$ letters (range 5-8) and a mean frequency $=9.3$ occurrences $/$ million (Dutch and French SUBTLEX databases). We excluded all translation equivalents, homonyms, interlingual homographs and homophones, and controlled for phonological and lexical neighbours. Stimuli were presented for a duration of $2500 \mathrm{~ms}$, preceded by a fixation cross presented in the centre of the screen for $300 \mathrm{~ms}$. Each scan run comprised 156 trials.

\section{Image acquisition and processing}

In a 3-T MRI Philips Achiva scanner, using a single-shot T2*-weighted EPI gradient-echo sequence $\left(\mathrm{TR} / \mathrm{TE} / \mathrm{Flip}=3000 \mathrm{~ms} / 35 \mathrm{~ms} / 90^{\circ}, \mathrm{FOV}=212 \times 230\right.$, matrix $=104 \times 105) 130$ dynamics were obtained over 22 axial slices (slice thickness $=4 \mathrm{~mm}$ ). Structural images were acquired using a T1-weighted, 3D gradient-echo pulse-sequence with TR $/ \mathrm{TE} / \mathrm{Flip}=12 \mathrm{~ms} / 3.75 \mathrm{~ms} / 10^{\circ}, \mathrm{FOV}=$ $240 \times 240$, matrix $=240 \times 240$, and slice thickness $=2 \mathrm{~mm}$. Data were analyzed using SPM5 (The Wellcome Institute of Neurology, London, UK). Functional images were spatially realigned, co-registered, spatially normalized to standard stereotactic MNI space, and smoothed with an isotropic 8-mm full-width at half-maximum Gaussian kernel.

\section{Results}

\section{Behavioural data}

Reaction times on the Simon task did not yield significant differences for simultaneous compared to sequential bilinguals ( $\mathrm{t}=-0.97, \mathrm{p}=0.341)$, although accuracy scores did $(\mathrm{t}=2.38, \mathrm{p}=0.027)$. Accuracy scores on the semantic categorization task showed a significant advantage of simultaneous over sequential bilinguals $(\mathrm{t}=6.65, \mathrm{p}=0.000)$. Reaction times on switches to 
French showed a significant difference for simultaneous compared to sequential bilinguals $(\mathrm{t}=-2.436, \mathrm{p}=0.023)$.

\section{Imaging data}

Within-group activations

On language conflict trials, simultaneous bilinguals showed activation in the caudate, precentral gyrus (BA6), and right inferior frontal gyrus (BA13); sequential bilinguals showed activation in the right superior frontal gyrus (BA8), bilateral inferior frontal gyrus (BA9), bilateral middle occipital gyrus (BA19), and right caudate.

Simon conflict trials for simultaneous bilinguals showed activation in the superior frontal gyrus (BA8), posterior (BA31) and central anterior (BA25) cingulate gyrus; in the sequential bilinguals showed activation in the superior parietal lobe (BA7), postcentral gyrus (BA2), superior frontal gyrus (BA8), and posterior cingulate gyrus (BA31).

Between-group activation

Relative to the simultaneous group on language conflict trials, sequential bilinguals showed activation in the superior parietal cortex (BA 7), precentral cortex (BA 6), anterior cingulate cortex (BA 32), and medial frontal cortex (BA 8). We found no specific activation for simultaneous bilinguals.

On Simon conflict trials, simultaneous compared to sequential bilinguals showed activation in the superior frontal cortex (BA 8), posterior (BA 8) and anterior (BA 25) cingulate cortex. Sequential compared to simultaneous bilinguals showed no significant activation.

\section{Discussion}

We identified distinct brain activations for each conflict type. For language conflict trials we found heightened activation in the inferior frontal cortex which is thought to be involved in response inhibition (Aron et al. 2004), in the superior parietal cortex (Liu et al. 2004), and in the caudate, which has been directly linked to language selection (Abutalebi \& Green 2007) and language control (Crinion et al. 2006). Simon conflict trials elicited activations in the superior frontal cortex which has been identified during $\mathrm{S}$ $\mathrm{R}$ conflicts in earlier studies (Früholz et al. 2010) and in the posterior cingulate cortex which is involved in spatial orienting (Vogt et al. 1992) or spatial target detection (Mesulam et al. 2001).

Interesting to note is that during both types of conflict trials we found activity in the anterior cingulate cortex which has been hypothesized to be responsible for conflict control and response inhibition in general (Liu et al. 2004) but also for stimulus-identity based stimulus-stimulus conflicts (van Veen \& Carter 2005). We cautiously interpret these data as suggesting that 
the anterior cingulate cortex may constitute a bridge between stimulusstimulus and stimulus-response conflict resolution and thus between language and cognitive control as a result of the more 'trained' use of this area during language conflict resolution in bilinguals.

\section{References}

Abutalebi, J., \& Green, D. 2007. Bilingual language production: The neurocognition of language representation and control. Journal of Neuroling., 20, 242-275.

Aron, A.R., Robbins, T.W., Poldrack, R.A. 2004. Inhibition and the right inferior frontal cortex. Trends Cogn. Sci. 8, 170-177.

Bialystok, E., Craik, F. I. M., Klein, R., \& Viswanathan, M. 2004. Bilingualism, aging, and cognitive control: Evidence from the Simon task. Psychology and Aging 19, 290-303.

Crinion, J., Turner, R., Grogan, A., Hanakawa, T., Noppeney, U., Devlin, J.T., Aso, T., Urayama, S., Fukuyama, H., Stockton, K., Usui, K., Green D.W., Price C.J. 2006. Language control in the bilingual brain. Science 312, 1537-1540.

Frühholz, S., Godde, B., Finke, M., Herrmann, M. 2010. Spatio-temporal brain dynamics in a combined stimulus-stimulus and stimulus-response conflict task. Neuroimage 54, 622-634.

Garbin, G., Sanjuan, A., Forna, C., Bustamante, J.C., Rodriguez-Pujadas, A., Belloch, V., Hernandez, M., Costa, A., \& Ávila, C. 2010. Bridging language and attention: Brain basis of the impact of bilingualism on cognitive control. Neuroimage 53, 1272-1278.

Liu, X., Banich, M.T., Jacobson, B.L., Tanabe, J.L. 2004. Common and distinct neural substrates of attentional control in an integrated Simon and spatial Stroop task as assessed by event-related fMRI. Neuroimage 22, 1097-1106.

Mesulam, M.M., Nobre, A.C., Kim, Y.H., Parrish, T.B., Gitelman, D.R. 2001. Heterogeneity of cingulate contributions to spatial attention. Neuroimage 13, 1065-1072.

Van Heuven, W.J.B., Schriefers, H., Dijkstra, T., \& Hagoort, P. 2008. Language conflict in the bilingual brain. Cerebral Cortex, 18, 2706-2716

Van Veen, V., Carter, C.S. 2005. Separating semantic conflict and response conflict in the Stroop task: a functional MRI study. Neuroimage 27, 497-504.

Vogt, B.A., Finch, D.M., Olson, C.R. 1992. Functional heterogeneity in cingulate cortex: the anterior executive and posterior evaluative regions. Cereb. Cortex 2, 435-443.

Ye, Z., \& Zhou, X. 2009. Executive control in language processing. Neuroscience and Biobehavioral Reviews 33, 1168-1177. 